\title{
Gaming on the Go: Examining the Resurgence and Growth of the Mobile and VR Games Markets During the Pandemic
}

\author{
Chengfeng Jiang
}

\begin{abstract}
Queens' University
17cj15@queensu.ca
\end{abstract}

\begin{abstract}
Due to the global financial crisis in 2020, businesses in various industries, including video gaming, had to make adjustment to adapt the sudden stop in their operations. This caused the gaming industry to capitalize on the opportunity. Due to the rise of the pandemic and the increasing demand for gaming consoles, the video game industry needed to respond swiftly. This was a great opportunity for mobile platforms to step up and provide an entertainment level that would not be feasible for the PC and console markets. This study explores the resurgence and growth of the video game market, through the various factors that have contributed to this growth. It also reviews the various trends that have affected the gaming industry, such as the rise of virtual reality (VR) and mobile gaming. This research concludes that aside from the rapid growth of gaming sector both in market size and value, the democratization, which means higher accessibility to more players, diversification of game producers, and technological upgrading are pivotal features of the development pattern of gaming industry during COVID-19.
\end{abstract}

Keywords: Mobile gaming, Video Games, Mobile gaming's democratizing, Virtual Reality (VR), Personal Computer (PC).

\section{INTRODUCTION}

The market of video games and virtual reality experienced a massive surge from the onset of the COVID-19 pandemic, standing out among other industries that had no choice but to ramp down as a result of widespread lock downs and stay-at-home orders. Simply put, everyone now has time to purchase a console or a gaming PC in order to spend their free time indoors. With the rest of the business industries scrambling to adjust to the sudden halt in movement, operations, and sale in the global market due to this crisis, the video gaming industry was able to capitalize on this set-up. Mobile gaming and virtual reality (VR) associated technology, one of the more notable submarkets in video games, also saw great resurgence as a consequence of the state of the world in 2020. With smart phones and VR headsets becoming almost as ubiquitous as a television or a refrigerator, the possibility of a larger and more democratized mobile games industry seems closer than ever before. With the world still in the middle of tackling the corona virus issue, mobile and VR gaming will likely experience unprecedented development and market growth on a level that will likely even outpace their PC and console counterparts. The video game market has experienced a resurgence over the past couple of years, largely due to the various factors that contributed to this growth. Since the pandemic which is expected to be long lasting will continuously engender substantial influence on economics and consuming behavior and is highly likely to reshape the dynamics and normality of video gaming industry, an exhaustive study regarding the development of game industry is crucial for us and is able to provide us opportunities to learn from the past and formulate guiding strategy for video games industry in the future.

\section{MOBILE GAMING AND DEMOCRATIZING VIDEO GAMES}

Within the multi-billion-dollar revenue of video gaming in 2020, a significant percentage and share of this revenue was attributed to mobile gaming. For example, according to the "Newzoo" analytic platform (Newzoo is a global leader in game and esports market research data analysis and expertise) latest projections 
for the global games market are based on their subscribers' data. In 2020, the games market is expected to generate revenues of 153.3 billion dollars, and has 9.3 percent of health year-of-year growth. The pandemic opened a market opportunity that proved instrumental in re-establishing the dominance of mobile gaming, and providing opportunities towards growing the games market as a whole[1]. Some critics may attempt to attribute the success of the mobile and VR games market to mere circumstance and coincidence, but the data surrounding these increased numbers suggest otherwise. First, there is the clear demand which was created by the COVID-19 pandemic, and likely led to a growth in video game sales regardless of context. However, the sheer exponential growth in the demand for video games, mobiles, consoles, or PC, demonstrated a need for the market to provide entertainment that was accessible and affordable [2][3]. Thus, the pandemic and the increased demand necessitated a swift and appropriate response from video game developers. Publishers and console manufacturers are both involved. People are more inclined to play games during fragmented spare times, hereby demand games to be fast-paced. This was a prime opportunity for the mobile market to step up and provide entertainment on a level that might not be feasible for the larger console and PC industries. In a sense, one can describe the growth of mobile and VR gaming as a reclamation of power in video games as a whole. Thus, the mobile market is contributing to a "democratization" of the video gaming industry that frees it from the control of the console and PC markets. But first, one must understand how mobile gaming became one of the pillars of the modern video game industry in the first place, and perhaps its history will help better inform the market of its eventual destination[8].

\section{CONTEXT AND EMERGENCE OF MOBILE GAMING}

The popularity of mobile gaming is predicated on its simple and user-friendly feature, and its role as a convenient alternative to mainstream console and PC games that might seem inaccessible to those living within lock-down who cannot afford a brand new Play Station 5 or a pre-built gaming computer. As an alternative to computer gaming, mobile gaming reduced the mechanisms that required extensive hardware capabilities and features needed to simply pay a quick game. In other words, it significantly reduced the effort and time necessary to allow consumers to play video games, introducing an unprecedented level of ease of access[2][3]. This led to the rise in popularity of a variety of games, from the classic "Tetris" to modern titles such as "Innersloth's Among Us" and "PlayerUnknown's Battlegrounds (PUBG)", which widened the market and audience of video games.]Generally speaking, mobile games allowed for almost anyone with a phone to quickly pass their time playing games[4][8]. It was clear that a significant portion of the video games market wanted mobile, portable, and compact game titles that featured easy-tounderstand control layouts and low-cost hardware. Notably, the mobile games market existed as early as 2002, with cellular phones sporting more hardware and computing power than ever before, but people have truly turned to it as a legitimate gaming device only in recent years[2][3]. In 2020, mobile game players logged in a global average of around 234 minutes a day. This corroborates the developed feature of mobile gaming that catered to the demand of the pandemic, and relegated the relevance and practicality of playing computer games[8]. However,it capitalized on the participatory features of PC and console technology developed as early as 2006 with Nintendo's Wii, and innovated on the stagnant features of extensive hardware and accessories that precluded the portability of video games[8]. Springing from this portable and compact feature that makes it accessible, the democratization of video games through mobile games followed[3] .

\section{MOBILE GAMING IMPORTANCE AND DEMOCRATIZATION}

The significance of "ease in access" in mobile gaming highlights another aspect in the deepening democratization of the industry. This manifests through more affordable price points for games, which widens the potential market and allows for increased consumer spending. augmented to the increasing revenues of spending consumers. For example, mobile gaming adopts free-to-play models that can be accessed through an internet browers or a downloadable application.In theory, this should entice potential customers to try the game out, thus increasing the popular coverage of the game itself[4]. During the start of pandemic, 15 million downloads both from the App Store and Google Play were recorded in one quarter, and further increased the following quarter with an additional 18.3 million downloads.Free downloads do not necessarily mean zero profits, as paid services may take the form of inapp purchases, in-game currency, or a premium version that allows for the removal of advertisements. Regardless, the "free" model led to a growth in game spending in 2020 that reached as high as 20.9 U.S[2], billion dollars, which projected increases lasting until 2023.With an audience and market that continually increases, especially during the span of lock down and unimpeded internet connectivity, the future of the market appears to center around the opportunities available to mobile gaming[6] 


\section{CHANGING DEMOGRAPHICS OF MOBILE GAMERS AND CREATORS}

Another factor which contributes to record-breaking growth within the mobile and VR gaming markets is the diversity of both the game developers and game players. This diversity is not only instrumental to the expansion of the games themselves, but also the transformation of the development and creative landscape in video games. The line between game players and game maker blurs thanks to a diversified change in the demographics of the market.Consumers of mobile gaming are transforming from a demographic that is highly structured and categorized by age and spending capabilities, to a diverse market which is unrestricted by profile limitations[7]. If video games were formerly marketed to a bracket of teenage to 35 years old or young adults , mobile gaming now attracts users beyond this categorization. In other words, the potential for mobile games appears unhindered by the usual targeting limitations inherent in the console and PC markets[3]. Aside from age, the industry may also capitalize in the demographic diversity of talent available to game development companies(Smith,2021). This means that there are more talented individuals for companies to hire, allowing for more minds to work on increasingly creative and fun games. In fact, some of these people might even be former game players themselves. Alternatively, the players may directly become involved with the development process through feedback. Again, this hearkens to the end of the distinction between game players and creators. As a culture of participative gaming is encouraged by this medium in video games, users become members of a gaming community that is highly involved in the creative process of developing game applications themselves[7][8]. One particular area where this concept thrives is virtual reality (VR). Within virtual reality, hyper-realistic visualizations, animations, and graphics in a real-time 3D platform are incorporated to the gaming experience[7]. Using mobile games as a springboard for their success, VR as an innovation also seems to be benefiting from the current status of the games market[6].

\section{VR AS INNOVATION AND THE "NEW OLD"}

The history and development of VR in video games dates back earlier than its emerging popularity. As a " new old" development in the history of the industry, its introduction dates back as early as 1950s with the launch of Telesphere Mask by Morton Heilig. Herein the impact of mobile gaming and the pandemic once again becomes instrumental in reintroducing this innovative technology in visualization and graphic images, to a market more conducive and responsive to its development and rise[3].Through its integration with the aforementioned appealing features of mobile gaming, VR technology looks forward to steadily growing in the market, and keeping relevance in the video games space[8] .

\section{INNOVATIONS IN VR TECHNOLOGY}

Originally, the hype of VR technology during its introduction in the early shift from 2D to 3D graphics died easily thanks to the limitations of computers at the time. However, due to the recent innovations of this technology in the modern context is founded on more stable ground as it incorporates the participative features of mobile gaming amidst the lock down. With social media platforms and gaming developers acquiring VR gaming companies such as Oculus in 2014, the profitability of the industry is emerging amidst the pandemic with a reported revenue from 1-29 million U.S. dollars in early 2020[2]. This revenue in the industry is projected to increase until 2023 with its continuous innovations such as developing affordable headsets that are compatible with most smart phones[6]. By simply mounting smart phones to these portable headsets, consumers are offered a taste of the VR experience at a fraction of the cost[6].

\section{CONCLUSION}

During the pandemic, the demand for the games market size has increased. As mobile gaming has become more democratized, it is provided an entertainment experience that is the larger consoles and PC markets might not have. As the technology is becoming more sophisticated with mobile gaming, and the most games have more reasonably priced are making the mobile gaming becomes more and more easy in access. This is exactly what gamers are looking for nowadays. Another key factor that contributes to the rapid growth of the VR and mobile gaming markets is the diversity of developers and game players. As game companies hire more talented people, some of whom are former gamers themselves. This diversity improve the transformation of the development and creative landscape in video games, and lead the gaming market to a healthy economic cycle. Part of the future of the gaming industry is now predicated on the development of mobile gaming and VR technology, which will likely continue to grow in the coming years. This growth seems almost guaranteed thanks to a democratized video game industry and technological developments that allow for accessible and affordable technology during the pandemic. Although issues related to the sustainability of mobile gaming and virtual reality remains relevant, the industry will continue to tweak its products in order to continuously deliver new content to the consumer. 


\section{REFERENCES}

[1]2020, M. W. T. (2020, October 1). The World's 2.7 Billion Gamers Will Spend \$159.3 Billion on Games in 2020; The Market Will Surpass \$200 Billion by 2023. Newzoo. https://newzoo.com/insights/articles/newzoogames-market-numbers-revenues-and-audience2020-2023/

[2]Feijoo, C., Gómez-Barroso, J. L., Aguado, J. M., \& Ramos, S. (2012). Mobile gaming: Industry challenges and policy implications. Telecommunications Policy, 36(3), 212-221. https://doi.org/10.1016/j.telpol.2011.12.004.

[3]Trevor, E. (2021). Encyclopedia of Video Games [3 volumes]: The Culture, Technology, and Art of Gaming. In M. J. P. Wolf (Ed.), Industry (2nd ed., pp. 314-319). Greenwood.

[4]Furini, Marco. (2007). Mobile Games: What to expect in the near Future. 93-95.

[5]Peter, K. (2014). The Routledge Companion to Video Game Studies (Routledge Media and Cultural Studies Companions). In M. J. P. Wolf \& B. Perron (Eds.), Violence (1st ed., pp. 345-352). Routledge.

[6]Noah Smith, N. S. (2021, February 4). Virtual reality is starting to see actual gains in gaming. The Washington Post. https://www.washingtonpost.com/videogames/2021/02/04/virtual-reality-future-games/.

[7]Wirman, H. (2009, September 15). On productivity and game fandom | Transformative Works and Cultures. Research Gate. https://journal.transformativeworks.org/index.php/t wc/article/view/145

[8]Young, C. (2021). Unity Production: Capturing the Everyday Game Maker Market. In Sotamaa O. \& Švelch J. (Eds.), Game Production Studies (pp. 141-158). Amsterdam: Amsterdam University Press. doi:10.2307/j.ctv1hp5hqw.10 